\title{
Editorials
}

\section{A public health approach to reducing health inequalities among adults with autism}

People with autism make up around 1\% of adults living in the community across the UK. ${ }^{1}$ Autism is a neurodevelopmental disorder which manifests from early childhood, with persistent deficits in social communication and social interaction across multiple contexts, along with restricted, repetitive patterns of behaviour, interests, or activities. The current guidance enables diagnosis of autism from level one to three based on the level of support needed to overcome the challenges posed by restrictive behaviours and social communication deficits. ${ }^{2}$

The health needs of adults with autism is of public health interest because this vulnerable population are socially disadvantaged and often 'hidden' from existing health and social care services." Autism is more prevalent in males, those without educational qualifications, and those living in social housing. ${ }^{1}$ People with autism with or without an intellectual disability (ID) are at higher risk of multimorbidity in the physical and mental health domains. ${ }^{3}$ Around $30 \%$ of adults with autism have comorbid ID, physical, and mental health conditions. ${ }^{4}$ Additionally, a recent study using Scotland's 2011 census data, found that adults with autism ( $n=6649$ ) can also experience sensory impairments, such as deafness/hearing loss $(14.1 \%, n=939)$ and blindness/sight loss (12.1\%, $n=807$ ), which can cause additional communication problems. ${ }^{4}$

While those with comorbid ID are more susceptible to physical health issues, ${ }^{5}$ those without ID are at higher risk of mental health problems, including higher than normal suicide rates. ${ }^{6}$ Adults with autism without ID die 12 years earlier, with suicide being the greatest cause of premature mortality. Therefore, this vulnerable population have significantly higher premature mortality rates when compared to the general population. Addressing the complex and disparate health needs of adults with autism requires innovative population based strategies to inform, enable, and empower the target group and its stakeholders.

\section{ACCESSING APPROPRIATE AND TIMELY HEALTH CARE}

Importantly, many of these adverse health outcomes are avoidable and can be addressed through appropriate levels of preventive health care, support, and lifestyle interventions. Tailoring the levels of severity

\section{"Annual Health Checks along with 'autism friendly practices ... can help meet the health needs of adults}

\section{with autism}

detailed in the Diagnostic and Statistical Manual of Mental Disorders (DSM-5) $)^{5}$ can be used to inform future interventions. It is important to address factors contributing to these adverse health outcomes in adults with autism. Evidence from childhood studies suggests that these include diet and/ or nutritional problems, social impairment, sedentary behaviours, emotional problems, and avoidance behaviours. ${ }^{8.9}$ As has been proposed in the NHS Long Term Plan, ${ }^{10}$ adults with autism may benefit from accessing an Annual Health Check (AHC), which are routinely offered to adults with a learning disability. ${ }^{11,12}$ People with ID share many of the primary health and social concerns adults with autism have. Despite challenges in delivery, the concept and mechanism of AHCs are considered an effective approach to identifying and managing a wide range of health conditions. ${ }^{13}$ Unfortunately, adults with autism, unless they have a comorbid ID, are not routinely offered an AHC because NHS bodies are not incentivised in the same way as supporting adults with ID. ${ }^{14}$

Additionally, adults with autism experience a range of barriers to appropriate and timely health care and support. These include issues that are intrinsic to the nature of autism Isensory impairment, for example, being unable to experience pain; communication difficulties; health literacy; anxiety; avoidance behaviours or lack of routine; inertia; neglect; and social isolation), ${ }^{15}$ but also insufficient reasonable adjustments that take into account the patient's specific needs. ${ }^{15}$

Organisationally, healthcare barriers include an inflexible approach to care settings, lack of autism leadership or champions in institutions, lack of clinical understanding of complexity of co-occurring physical and mental health issues, and diagnostic overshadowing. ${ }^{14}$ Corporate health care is divided into rigid specialties from commissioning and resource perspectives, leading to gaps in the system. Thus, complex patients with multimorbidity are highly prone to fall through these gaps. These barriers contribute to $74 \%$ of adults with autism reporting that they experience difficulties in accessing health care, and $88 \%$ felt that health professionals failed to understand their health needs. ${ }^{13}$ Reduced access to timely and appropriate health care contributes to the inequalities experienced by adults with autism. This represents a significant burden on society and health and social care services, costing the UK economy $€ 32$ billion per annum due to poor physical and/or mental health, and loss of earnings. ${ }^{14}$

\section{REDUCING HEALTH INEQUALITIES}

The Westminster Commission on Autism ${ }^{13}$ made an overwhelming case for preventative health care through $\mathrm{AHC}$ for adults with autism. This is supported by national legislation (such as, the Autism Act 2009), national policy, and guidance, such as those from the National Institute for Health and Care Excellence (NICE), ${ }^{16}$ which support the need to identify and manage people with autism's unmet health needs. The NHS Long Term Plan recently committed to the introduction of a specific $\mathrm{AHC}$ for people with autism, which will be extended more widely if successful. ${ }^{11}$ In addition, there is ongoing consultation on the need and type of autism training in all statutory public sectors.

While this is encouraging, some of the above barriers may continue to persist and influence the levels of uptake of preventative health care and support. These barriers make it challenging to support those with autism in a general practice, especially due to the wide spectrum of presentation and range of abilities. ${ }^{17}$ Consequently, GPs would benefit from endorsing the Autism Patient Charter la framework developed by RCGP and Autism Alliance to make primary care practices autism friendly. It provides five broad areas staff awareness, communication, reasonable adjustments, environment, and behaviour management - for practices to consider in relation to supporting people with autism) and implementing the RCGP Autistic Spectrum disorder toolkit. ${ }^{18}$ Such a toolkit could inform a range of issues including support in the delivery of reasonable adjustments, access to formal autism training, and overcoming issues such as diagnosis and clarity around 
referral pathways. ${ }^{19}$

Additionally, the development of an anonymous national primary care autism register ${ }^{14}$ can help overcome the 'hidden' nature of autism. Adopting an autism register can help target those who may benefit most from healthcare interventions, especially if the DSM- 5 suggested levels of autism model is used. However, some adults with autism may not welcome this approach because of potential negative stigma in areas such as employment.

\section{PUBLIC HEALTH APPROACHES}

Annual Health Checks along with autism friendly' practices with increased awareness among professionals, the provision of reasonable adjustments, and clear information, can help meet the health needs of adults with autism holistically. ${ }^{10}$ In addition, public health and health promotion teams in local authorities, social care, current autism charities, and health bodies, are well placed for developing alternative forms of health care and support (complementary to AHCs), which could potentially help overcome some of the obstacles that are intrinsic to autism. For example, the utilisation of existing budgets could help the delivery of support outside of traditional NHS settings, which may include exploring social prescribing opportunities.

A co-created 'health interview' to help adults with autism overcome emotional, communication, and sensory impairments, may reduce barriers seen in formal NHS settings. To be effective, these should be combined with the availability of AHCs. Developing, harnessing, and the testing of new technologies in health and social care, may further alleviate high levels of health needs among this population; for example, delivering a virtual consultation at the person's home using video-based web tools.

Finally, prior research has focused on childhood populations with a paucity of research on the health needs of adults with autism and a lack of direct health assessments, particularly in more rural environments. Further research is needed to understand the health needs of this vulnerable population across the life course to guide the development of future interventions to meet the health needs of adults with autism.

\section{CONCLUSION}

To maximise the potential benefits of AHCs for adults with autism, it is necessary to develop more holistic and collaborative ways of working with other bodies, such as adult social care and public health. This may be achieved by co-creating public health approaches, such as social prescribing or additional 'health interviews', which are offered outside of NHS settings, to help overcome some of the barriers to accessing timely and appropriate health care. This may help alleviate some of the individual and healthcare pressures. Additionally, working collaboratively with wider stakeholders will help engage with the 'hidden majority' of adults with autism (those who remain unknown to existing services), which will improve the effectiveness of AHCs.

\section{Richard A Sharpe,}

Advanced Public Health Specialist, Cornwall Council, Truro, Cornwall. Senior Honorary Research Fellow. European Centre for Environment and Human Health, College of Medicine and Health, University of Exeter, Truro, Cornwall.

\section{Whitney Curry,}

Advanced Public Health Practitioner, Cornwall Council, Truro, Cornwall.

\section{Rachel Brown,}

Psychologist, Spectrum, Truro, Cornwall.

\section{REFERENCES}

1. Brugha TS, McManus S, Bankart J, et al. Epidemiology of autism spectrum disorders in adults in the community in England. Arch Gen Psychiatry 2011; DOI: 10.1001/ archgenpsychiatry.2011.38.

2. American Psychiatric Association (APA). Diagnostic and statistical manual of mental disorders, fifth edition (DSM-5). Arlington, VA: APA, 2013.

3. BMA. Recognising the importance of physical health in mental health and intellectual disability. Achieving parity of outcomes. London: BMA, 2014

4. Rydzewska E, Hughes-McCormack LA, Gillberg C, et al. Prevalence of long-term health conditions in adults with autism: observational study of a whole country population. BMJ Open 2018; DOI: 10.1136/bmjopen-2018-023945.

5. Kinnear D, Morrison J, Allan L, et al. Prevalence of physical conditions and multimorbidity in a cohort of adults with intellectual disabilities with and without Down syndrome: crosssectional study. BMJ Open 2018; DOI: 10.1136/ bmjopen-2017-018292.

6. Zahid S, Upthegrove R. Suicidality in autistic spectrum disorders: a systematic review. Crisis 2017; 38(4): 237.

7. Hirvikoski T, Mittendorfer-Rutz E, Boman M, et al. Premature mortality in autism spectrum disorder. Br J Psychiatry 2016; DOI: 10.1192/bjp. bp.114.160192

8. Ranjan S, Nasser JA. Nutritional status of individuals with autism spectrum disorders: do we know enough? Adv Nutr 2015; DOI: 10.3945/ an. 114.007914 .

9. Jones RA, Downing K, Rinehart NJ, et al. Physical activity, sedentary behavior and their correlates in children with autism spectrum disorder: a systematic review. PLoS One 2017; DOI: 10.1371/ journal.pone.0172482.

10. NHS England. The NHS Long Term Plan. 2019.

\section{ADDRESS FOR CORRESPONDENCE}

\section{Rohit Shankar}

Cornwall Partnership NHS Foundation Trust, Truro Cornwall TR4 9LD, UK.

\section{Email: rohit.shankaranhs.net}

\section{Rohit Shankar,}

Consultant in Adult Developmental Neuropsychiatry, Cornwall Partnership NHS Foundation Trust, Truro Cornwall, UK. Honorary Associate Clinical Professor (Senior Clinical Lecturer) University of Exeter Medical School Truro, Cornwall.

\section{Funding}

This article was supported by Cornwall Public Health function. No external funding was obtained.

\section{Provenance}

Freely submitted; externally peer reviewed.

\section{Competing interests}

Rohit Shankar has received institutional and research support, and personal fees from LivaNova, Union Chimique Belge (UCB), Eisai, Veriton Pharma, Bial, and Desitin, outside of the submitted work. All other authors have declared no competing interests.

DOI: https://doi.org/10.3399/bjgp19X706133

https://www.longtermplan.nhs.uk/wp-content/ uploads/2019/08/nhs-long-term-plan-version1.2.pdf (accessed 20 Sep 2019).

11. NHS England. Annual Health Checks. 2019. https://uww.england.nhs.uk/learning-disabilities/ improving-health/annual-health-checks laccessed 20 Sep 2019).

12. Robertson J, Hatton C, Emerson E, Baines S. The impact of health checks for people with intellectual disabilities: an updated systematic review of evidence. Res Dev Disabil 2014; DOI: 10.1016/j.ridd.2014.06.007.

13. The Westminster Commission on Autism. A spectrum of obstacles: an inquiry into access to healthcare for autistic people. 2016. https:// westminsterautismcommission.files.wordpress. com/2016/03/ar1011_ncg-autism-reportjuly-2016.pdf (accessed 10 Oct 2019).

14. Buckley $C$. Making your practice autism friendly. InnovAiT 2017; 10(6): 327-331.

15. Thye MD, Bednarz HM, Herringshaw AJ, et al. The impact of atypical sensory processing on social impairments in autism spectrum disorder. Dev Cogn Neurosci2017; DOI: 10.1016/j. dcn.2017.04.010.

16. National Institute for Health and Care Excellence. Autism spectrum disorder in adults: diagnosis and management. Clinical guideline [CG142] 2016. https://www.nice.org.uk/guidance/cg142 laccessed 11 Oct 2019).

17. Buckley C. Autism in adults. InnovAiT2017; 10(6): 319-326.

18. Royal College of General Practitioners. Autistic Spectrum disorder toolkit. https://www.rcgp.org uk/clinical-and-research/resources/toolkits/asdtoolkit.aspx (accessed 10 Oct 2019).

19. Unigwe, S, Buckley C, Crane L, et al. GPs' confidence in caring for their patients on the autism spectrum: an online self-report study. Br J Gen Pract 2017; DOI: https://doi.org/10.3399/ bjgp17X690449. 